\title{
Improving the effectiveness of home parenteral nutrition clinic - the Leeds proforma
}

\author{
J. M. Wood, H. Lynch, S. Rahmani, J. Scantlebury, C. F. Donnellan and D. A. Burke \\ Leeds Enteral and Parenteral Nutrition Team, Leeds General Infirmary, Leeds, LS1 3EX, UK
}

Current ESPEN guidelines recommend that HPN patients are monitored on a 3-monthly basis, although this may vary depending on the stability of the patient. Recent increases in numbers of home parenteral nutrition (HPN) and intravenous fluid-dependent patients under the care of the Leeds Enteral And Parenteral (LEAP) Nutrition Team have resulted in clinic numbers rising from an average of 8 per clinic to 16 per clinic and thus the need for quicker and more comprehensive access to relevant information.

In order to improve the quality of patient review, over the last 12 months we have introduced a proforma for each home-care patient detailing various categories of information which is available for all members of the nutrition team via a central server.

Each proforma contains information regarding:

1. Detailed colorectal surgical history combined with a pictorial representation of current anatomy.

2. Co-morbidities.

3. Current and previous intravenous catheter history.

4. Current medication.

5. Scan results, in particular DEXA scans.

6. Current and previous clinic annotations.

7. Changes made after review of blood tests, taken in clinic.

8. BANS history.

To audit the efficacy, we analysed various categories of the proforma for patients who have been receiving HPN for 2 years or more (n 17).

ESPEN guidelines recommends that HPN patients have bone mineral density assessed by DEXA scan on a yearly basis. However, local policy states that DEXA scans need only be performed every 2 years.

1. Forty-seven percent of pts had DEXA scans before introduction of proforma.

2. Hundred percent of pts who had DEXA scans 12 months after introduction $100 \%$ $P<0.008$ (McNemar's).

3. Forty-seven percent of patients were registered on BANS before introduction of proforma.

4. Hundred percent were registered on BANS in the 12 months after introduction $P<0.008$ (McNemar's).

5. All patients were sent appointments for clinics with the longest interval at 3 months.

The introduction of the proforma has allowed the team access to valuable information in these complex patients with multiple volumes of notes. There is now a clear trail for decisions taken with a significant improvement in care where measurable outcomes were possible. 